\title{
Research on electromagnetic wave propagation in fractional space
}

\author{
Zhang Hongbo ${ }^{1, a}$ and Yang Zhongshu ${ }^{1, b^{*}}$ \\ ${ }^{1}$ Naval aeronautical engineering application, Beijing, 100071, China \\ azhanghongbo90@163.com, byangzhongshu01@163.com
}

Keywords: Fractional derivatives; Fractional space; Fractal media; Fractional Maxwell's equations. Abstract. In this paper, a generalization of differential electromagnetic equations in fractional space is provided. These equations can describe the behavior of electric and magnetic fields in any fractal media. The time evolution of the fractional electromagnetic waves by using the time fractional Maxwell's equations in fractional space has been investigated. Theoretical analysis shows that the amplitude variations of the general plane wave solution not only is related to Bessel functions, but also reveals an algebraic decay, at asymptotically large times.

\section{Introduction}

In recent years, fractional calculus has attracted many researchers successfully in different disciplines of science and engineering. One of the main advantages of the fractional calculus is that the fractional derivatives provide a superior approach for the description of memory and hereditary properties of various materials and processes [1-3]. Applications of fractional calculus in the field of mathematics, chemistry, physical, biology and engineering have gained considerable achievement and many significant results were obtained [4-7]. More and more researchers are finding that numerous important dynamical problems exhibit fractional order behavior which may vary with space and time. This fact illustrates that fractional calculus is a natural candidate to provide an effective mathematical framework for the description of complex dynamical problems. Some materials and media that have electromagnetic memory properties can be expressed by fractional nonlocal formalism. Therefore the generalized fractional Maxwell's equations can give us many new models that can be used in complex systems.

In the last few decades, there has been considerable interest in the study of physical description of confinement in low dimensional systems assuming a fractional dimension of the space [8-10]. The idea to take place of the real confining structure by a space, where the measure of confinement or anisotropy is given by non-integer dimension, was proposed in [11]. Such confinement may be described in low dimensional systems which can have various degrees of confinement in various orthogonal directions. In [12] fractal structures had been discussed within the fractional dimensional space approach. Axiomatic basis for the idea of fractional space for two-spatial coordinate space was given in [13], and this work was extended to orthogonal coordinate space in [8]. Stillinger [13] studied a formalism for constructing a generalization of an integer dimensional Laplacian operator into a non-integer dimensional space. Engheta $[14,15]$ introduced an early investigation of fractional solution of wave equation. Zubair and Mughal [16] developed an exact solution of the cylindrical wave equation for electromagnetic field in fractional dimensional space.

The aim of this work is to study the time evolution of the fractional electromagnetic waves by means of the time fractional Maxwell's equations in fractional space. For this purpose, in the following section we review electromagnetic theory.

\section{Fractional space generalization of differential Maxwell's equations}

The Maxwell's equations are the fundamental equations describing the property of electric and magnetic fields. The basic classical Maxwell's equations in differential form as following:

$\nabla \cdot \boldsymbol{D}=\rho_{v}$

$\nabla \cdot \boldsymbol{B}=0$ 
$\nabla \times \boldsymbol{E}=-\frac{\partial \boldsymbol{B}}{\partial t}$

$\nabla \times \boldsymbol{H}=\boldsymbol{J}+\frac{\partial \boldsymbol{D}}{\partial t}$

the quantities are dealt with:

$\boldsymbol{E}=$ electric field intensity $(\mathrm{V} / \mathrm{m})$;

$\boldsymbol{H}=$ magnetic field intensity $(A / m)$;

$\boldsymbol{D}=$ electric flux density $\left(C / \mathrm{m}^{2}\right)$;

$\boldsymbol{B}=$ magnetic flux density $\left(W / \mathrm{m}^{2}\right)$;

$\boldsymbol{J}=$ electric current density $\left(A / \mathrm{m}^{2}\right)$;

$\rho_{v}=$ electric charge density $\left(C / \mathrm{m}^{3}\right)$,

with $\boldsymbol{B}=\mu \boldsymbol{H}$ and $\boldsymbol{D}=\varepsilon \boldsymbol{E}$, where $\mu$ and $\varepsilon$ are permeability and permittivity of the medium, respectively. All of these quantities are functions of space variables $x, y, z$ and time $t$.

We may simply introduce the generalized form of Maxwell's equations in $D$-dimensional fractional space, more detailed introduction can be found in the Ref. [17]. The differential form of Maxwell's equations in far-field region in fractional space are given by:

$\nabla_{D} \cdot \boldsymbol{D}=\rho_{v}$

$\nabla_{D} \cdot \boldsymbol{B}=0$

$\nabla_{D} \times \boldsymbol{E}=-\frac{\partial \boldsymbol{B}}{\partial t}$

$\nabla_{D} \times \boldsymbol{H}=\boldsymbol{J}+\frac{\partial \boldsymbol{D}}{\partial t}$

where Del operator $\nabla_{D}$ in fractional space is expressed as:

$\nabla_{D}=\left(\frac{\partial}{\partial x}+\frac{1}{2} \frac{\alpha_{1}-1}{x}\right) \hat{x}+\left(\frac{\partial}{\partial y}+\frac{1}{2} \frac{\alpha_{2}-1}{y}\right) \hat{y}+\left(\frac{\partial}{\partial z}+\frac{1}{2} \frac{\alpha_{3}-1}{z}\right) \hat{z}$

where the parameters $0<\alpha_{1} \leq 1,0<\alpha_{2} \leq 1$, and $0<\alpha_{3} \leq 1$ are used to describe the measure distribution of space where each one is acting independently on a single coordinate and the total dimension of the space is $D=\alpha_{1}+\alpha_{2}+\alpha_{3}$. Obviously, if we set $\alpha_{1}=\alpha_{2}=\alpha_{3}=1$, the fractional Del operator $\nabla_{D}$ reduces to the classical Del operator $\nabla$.

Now, introducing the potentials, vector $\boldsymbol{A}$ and scalar $\varphi$

$\boldsymbol{B}=\nabla_{D} \times \boldsymbol{A}$

$\boldsymbol{E}=-\nabla_{D} \varphi-\frac{\partial \boldsymbol{A}}{\partial t}$

Then we obtain the following differential equations for the potentials:

$$
\begin{aligned}
& \nabla_{D}^{2} \varphi-\mu \varepsilon \frac{\partial^{2} \varphi}{\partial t^{2}}=-\frac{\rho_{v}}{\varepsilon} \\
& \nabla_{D}^{2} \boldsymbol{A}-\mu \varepsilon \frac{\partial^{2} \boldsymbol{A}}{\partial t^{2}}=-\mu \boldsymbol{J}
\end{aligned}
$$

To write the generalization of Maxwell's equations (5)-(8) in the fractional with respect to $t$, by replacing the time derivative with a fractional derivative of order $\alpha(0<\alpha \leq 1)$ namely:

$\nabla_{D} \cdot \boldsymbol{D}=\rho_{v}$

$\nabla_{D} \cdot \boldsymbol{B}=0$

$\nabla_{D} \times \boldsymbol{E}=-\frac{1}{\xi^{1-\alpha}} \frac{\partial^{\alpha} \boldsymbol{B}}{\partial t^{\alpha}}$ 
$\nabla_{D} \times \boldsymbol{H}=\boldsymbol{J}+\frac{1}{\xi^{1-\alpha}} \frac{\partial^{\alpha} \boldsymbol{D}}{\partial t^{\alpha}}$

and Eq.(10) and (11) become

$\boldsymbol{B}=\nabla_{D} \times \boldsymbol{A}$

$\boldsymbol{E}=-\nabla_{D} \varphi-\frac{1}{\xi^{1-\alpha}} \frac{\partial^{\alpha} \boldsymbol{A}}{\partial t^{\alpha}}$

In above equations the fractional derivative of order $\alpha, n-1<\alpha \leq n, n \in N$ is defined in the Caputo sense [18]:

$D_{*}^{\alpha} f(t)=\left\{\begin{array}{lr}\frac{d^{n} f(t)}{d t^{n}}, & \alpha=n \in N ; \\ \frac{1}{\Gamma(n-\alpha)} \int_{0}^{t} \frac{f^{(n)}(T)}{(t-T)^{\alpha-n+1}} d T, & 0 \leq n-1<\alpha<n .\end{array}\right.$

where $\Gamma(\cdot)$ denotes the Gamma function. For $\alpha=n, n \in N$ the Caputo fractional derivative is defined as the standard derivative of order $\alpha$. Also, we should note that the arbitrary quantity $\xi$ with dimension of [second] is to ensure all quantities have correct dimensions. Now we can get the corresponding time fractional wave equations for the potentials

$\nabla_{D}^{2} \varphi-\mu \varepsilon \frac{1}{\xi^{2(1-\alpha)}} \frac{\partial^{2 \alpha} \varphi}{\partial t^{2 \alpha}}=-\frac{\rho_{v}}{\varepsilon}$

$\nabla_{D}^{2} \boldsymbol{A}-\mu \varepsilon \frac{1}{\xi^{2(1-\alpha)}} \frac{\partial^{2 \alpha} \boldsymbol{A}}{\partial t^{2 \alpha}}=-\mu \boldsymbol{J}$

If $\rho_{v}=0$ and $\boldsymbol{J}=0$, we have the homogeneous fractional differential equations

$\nabla_{D}^{2} \varphi-\mu \varepsilon \frac{1}{\xi^{2(1-\alpha)}} \frac{\partial^{2 \alpha} \varphi}{\partial t^{2 \alpha}}=0$

$\nabla_{D}^{2} \boldsymbol{A}-\mu \varepsilon \frac{1}{\xi^{2(1-\alpha)}} \frac{\partial^{2 \alpha} \boldsymbol{A}}{\partial t^{2 \alpha}}=0$

In above equations, $\nabla_{D}^{2}$ is the Laplacian operator in $D$-dimensional fractional space and is defined as follows

$\nabla_{D}^{2}=\frac{\partial^{2}}{\partial x^{2}}+\frac{\alpha_{1}-1}{x} \frac{\partial}{\partial x}+\frac{\partial^{2}}{\partial y^{2}}+\frac{\alpha_{2}-1}{y} \frac{\partial}{\partial y}+\frac{\partial^{2}}{\partial z^{2}}+\frac{\alpha_{3}-1}{z} \frac{\partial}{\partial z}$

Eq.(23) and Eq.(24) are the same form, we can write the fractional equations in the following compact form

$\left[\frac{\partial^{2}}{\partial x^{2}}+\frac{\alpha_{1}-1}{x} \frac{\partial}{\partial x}\right] Z(x, t)-\mu \varepsilon \frac{1}{\xi^{2(1-\alpha)}} \frac{\partial^{2 \alpha} Z(x, t)}{\partial t^{2 \alpha}}=0$

where $Z(x, t)$ represents both $\boldsymbol{A}$ and $\varphi$. Analytical solutions of Eq.(26) can be obtained by using separation of variables to get the fields inside the fractional space. We consider

$Z(x, t)=u(x) v(t)$

and the resulting ordinary differential equations are obtained as follows

$\left[\frac{d^{2}}{d x^{2}}+\frac{\alpha_{1}-1}{x} \frac{d}{d x}-\gamma_{x}^{2}\right] u(x)=0$

$\left[\frac{d^{2 \alpha}}{d t^{2 \alpha}}-\frac{\gamma_{x}^{2}}{\mu \varepsilon} \xi^{2(1-\alpha)}\right] v(t)=0$

where $\gamma_{x}$ is known as wave constants in the $x$ direction and will be determined using boundary 
conditions. Let $\Omega^{2}=\frac{\gamma_{x}^{2}}{\mu \varepsilon} \xi^{2(1-\alpha)}$, then Eq.(29) will be

$\left[\frac{d^{2 \alpha}}{d t^{2 \alpha}}-\Omega^{2}\right] v(t)=0$

The solution of Eq.(30) may be

$v(t)=E_{2 \alpha}\left(\Omega^{2} t^{2 \alpha}\right)$

where $E_{\alpha}(z)=\sum_{k=0}^{\infty} \frac{z^{k}}{\Gamma(1+\alpha k)}$ is one parameter Mittag-Leffler function.

Let $a=\alpha_{1}-1$, Eq.(28) can be written as

$\left[x \frac{d^{2}}{d x^{2}}+a \frac{d}{d x}-\gamma_{x}^{2} x\right] u(x)=0$

Eq.(32) is reducible to Bessel's equation by substituting $u(x)=x^{n} \eta(x)$ as follows:

$\left[x^{2} \frac{d^{2}}{d x^{2}}+x \frac{d}{d x}-\gamma_{x}^{2} x^{2}-n^{2}\right] \eta(x)=0$

where $n=\frac{|1-a|}{2}$, the solution of Bessel's equation in Eq.(33) is given as [19]:

$\eta(x)=C_{1} J_{n}\left(-j \gamma_{x} x\right)+C_{2} Y_{n}\left(-j \gamma_{x} x\right)$

where $J_{n}(x)=\sum_{k=0}^{\infty} \frac{(-1)^{k}}{k ! \Gamma(k+n+1)}\left(\frac{x}{2}\right)^{2 k+n}$ is referred to the Bessel function of the first kind of order $n$ and $Y_{n}(x)=\frac{J_{n}(x) \cos (n \pi)-J_{-n}(x)}{\sin (n \pi)}$ as the Bessel function of the second kind of order $n . C_{1}$ and $C_{2}$ are constant coefficients.

Finally, the solution of Eq.(28) becomes

$u(x)=x^{n_{1}}\left[C_{1} J_{n_{1}}\left(-j \gamma_{x} x\right)+C_{2} Y_{n_{1}}\left(-j \gamma_{x} x\right)\right]$

where $n_{1}=1-\frac{\alpha_{1}}{2}$.

Substituting Eq.(35) and Eq.(31) into Eq.(27), we obtain the analytical solutions of Eq.(26) as follows $Z(x, t)=x^{n_{1}}\left[C_{1} J_{n_{1}}\left(-j \gamma_{x} x\right)+C_{2} Y_{n_{1}}\left(-j \gamma_{x} x\right)\right] E_{2 \alpha}\left(\Omega^{2} t^{2 \alpha}\right)$

\section{Asymptotic behavior of the solution}

In contrast with the exponential decay of the usual standard form of the equations, the algebraic decay of the solutions of the fractional equations in fractional space is most important effect of the fractional derivative in the typical fractional equations. We consider the integral form for the Mittag-Leffler function to describe this algebraic decay. The asymptotic expansion of $E_{\alpha}(z)$ based on the integral representation in the form [20]:

$E_{\alpha}(z)=\frac{1}{2 \pi j} \int_{Y} \frac{t^{\alpha-1} e^{t}}{t^{\alpha}-z} d t$

where $\operatorname{Re}(\alpha)>0, \alpha, z \in C$ and the path of integration $Y$ is a loop starting and ending at $-\infty$ and encircling the circular disk $|t| \leq|z|^{\frac{1}{\alpha}}$ in the positive sense $|\arg t|<\pi$ on $Y$. Eq.(37) can be used to obtain the asymptotic expansion of the Mittag-Leffler function at infinity. According to the property of Mittag-Leffler function, we can obtain the following cases. 
If $0<\alpha<2$ and $\theta$ is a real number such that $\frac{\pi \alpha}{2}<\theta<\min [\pi, \pi \alpha]$, then for $N^{\prime} \in N, N^{\prime} \neq 1$, there holds asymptotic expansion as follows:

$E_{\alpha}(z)=\frac{1}{\alpha} z^{\frac{1-\beta}{\alpha}} e^{z^{\frac{1}{\alpha}}}-\sum_{r=1}^{N^{\prime}} \frac{1}{\Gamma(1-\alpha r)} \frac{1}{z^{r}}+O\left[\frac{1}{z^{N^{\prime}+1}}\right]$

as $|z| \rightarrow \infty,|\arg t| \leq \theta$ and

$E_{\alpha}(z)=-\sum_{r=1}^{N^{\prime}} \frac{1}{\Gamma(1-\alpha r)} \frac{1}{z^{r}}+O\left[\frac{1}{z^{N^{\prime}+1}}\right]$

as $|z| \rightarrow \infty, \theta \leq|\arg t| \leq \pi$. In our case, $z=\Omega^{2} t^{2 \alpha}$ then

$E_{2 \alpha}\left(\Omega^{2} t^{2 \alpha}\right) \approx-\frac{1}{\Gamma(1-2 \alpha)} \frac{1}{\Omega^{2} t^{2 \alpha}}$

Substituting Eq.(40) into Eq.(36), we have

$Z(x, t) \approx-\frac{C_{1}}{\Gamma(1-2 \alpha)} \frac{x^{n_{1}} J_{n_{1}}\left(-j \gamma_{x} x\right)}{\Omega^{2} t^{2 \alpha}}-\frac{C_{2}}{\Gamma(1-2 \alpha)} \frac{x^{n_{1}} Y_{n_{1}}\left(-j \gamma_{x} x\right)}{\Omega^{2} t^{2 \alpha}}$

From the result of Eq.(41), we can achieve the asymptotic for the electromagnetic wave equation which represents a general plane wave solution with algebraic time-decaying amplitude. In other word fractional differentiation with respect to time can be explained an existence of memory effects which correspond to intrinsic dissipation in our system.

\section{An example}

For the case $\alpha=\frac{1}{4}$, we have

$v(t)=E_{\frac{1}{2}}\left(\Omega^{2} \sqrt{t}\right)=e^{\Omega^{4} t}\left(1+\operatorname{erf}\left(\Omega^{2} \sqrt{t}\right)\right)=e^{\Omega^{4} t} \operatorname{erfc}\left(-\Omega^{2} \sqrt{t}\right)$

where $\operatorname{erfc}$ denotes the complimentary error function and the error function is given by

$\operatorname{erf}(z)=\frac{2}{\sqrt{\pi}} \int_{0}^{z} e^{-t^{2}} d t, \operatorname{erfc}(z)=1-\operatorname{erf}(z), \quad z \in C$

For large values of $z$, the complimentary error function can be approximated as

$\operatorname{erfc}(z) \approx \frac{1}{\sqrt{\pi} z} e^{-z^{2}}$

Substituting Eq.(42) into Eq.(36), we get

$$
Z(x, t)=x^{n_{1}}\left[C_{1} J_{n_{1}}\left(-j \gamma_{x} x\right)+C_{2} Y_{n_{1}}\left(-j \gamma_{x} x\right)\right] e^{\Omega^{4} t} \operatorname{erfc}\left(-\Omega^{2} \sqrt{t}\right)
$$

At asymptotically large times, and using Eq.(44), we obtain

$$
Z(x, t) \approx-x^{n_{1}}\left[C_{1} J_{n_{1}}\left(-j \gamma_{x} x\right)+C_{2} Y_{n_{1}}\left(-j \gamma_{x} x\right)\right] \frac{1}{\sqrt{\pi} \Omega^{2} \sqrt{t}}
$$

For this case, the solution represents general plane wave with time decaying amplitude.

\section{Summary}

The asymptotic behavior of Mittag-Leffler functions plays a significant role in understanding of the solutions of different problems of physic connected with fractional phenomena that occur in fractional space. We have discussed the time evolution of the fractional electromagnetic waves in fractional space by using the time fractional Maxwell's equations. We pointed that the amplitude of the general electromagnetic plane wave is described by Bessel functions, and it also exhibits an algebraic decay with time increasing in our system. 


\section{References}

[1] V.V. Anh, J.M. Angulo, M.D. Ruiz-Medina, Diffusion on multifractals, Nonlinear Analysis 63 (2005) e2053-e2056.

[2] W. Chen, A speculative study of 2/3-order fractional Laplacian modeling of turbulence: Some thoughts and conjectures, Chaos 16 (2006) 023126.

[3] H.G. Sun, W. Chen, H. Sheng, Y.Q. Chen, On mean square displacement behaviors of anomalous diffusions with variable and random order, Physics Letter A 374 (2010) 906-910.

[4] M. Ichise, Y. Nagayanagi, T. Kojima, An analog simulation of noninteger order transfer functions for analysis of electrode process, Journal of Electroanalytical Chemistry 33 (1971) 253- 265.

[5] H.H. Sun, A.A. Abdlwahad, B. Onaral, Linear approximation of transfer function with a pole of fractional order, IEEE Transactions on Automatic Control 29 (1984) 441-444.

[6] O. Heaviside, Electromagnetic Theory, Chelsea, New York, 1971.

[7] R.C. Koeller, Application of fractional calculus to the theory of viscoelasticity, Journal of Applied Mechanics 51 (1984) 299-307.

[8] C. Palmer, P.N. Stavrinou, Equation of motion in a non-integer dimension space, Journal of Physics A 37 (2004) 6987-7003.

[9] V. E. Tarasov, Electromagnetic fields on fractals, Modern Physics Letter A 21(20) (2006) 15871600.

[10] D. Baleanu, A.K. Golmankhaneh, A.K. Golmankhaneh, On electromagnetic field in fractional space, Nonlinear Analysis: Real Word Applications 11(1) (2010) 288-292.

[11] S. Muslih, D. Baleanu, Fractional multipoles in fractional space, Nonlinear Analysis: Real Word Applications 8 (2007) 198-203.

[12] B. Mandelbrot, The Fractal Geometry of Nature, W.H. Freeman, Now York, 1983.

[13] F.H. Stillinger, Axiomatic basis for spaces with non-integer dimension, Journal of Mathematical Physics 18(6) (1977) 1224-1234.

[14] N. Engheta, Use of fractional integration to propose some 'fractional' solutions for the scalar Helmholtz equation, Progress In Electromagnetic Research 12 (1996) 107-132.

[15] N. Engheta, On the role of fractional calculus in electromagnetic theory, IEEE Antennas and Propagation Magazine 39(4) 35-46 1997.

[16] M. Zubair and M. J. Mughal, An exact solution of the cylindrical wave equation for electromagnetic field in fractional dimensional space, Progress In Electromagnetic Research 114 (2011) 443-455.

[17] M. Zubair and M. J. Mughal, Differential electromagnetic equations in fractional space, Progress In Electromagnetic Research 114 (2011) 255-269.

[18] I. Podlubny, Fractional Differential Equations, Academic press, 1999, pp. 60-101.

[19] A.D. Polyanin, V.F. Zaitsev, Handbook of Exact Solution for Ordinary Differential Equations, second ed. CRC Press, Boca Raton, New York, 2003, pp.34-87.

[20] H.J. Hauhold, A.M. Mathai, P.K. Saxena, Mittag-Leffler Functions and Their Applications, Journal of Applied Mathematics 2011 (2011) 298628. 